\title{
A Novel Technique for Achieving the Approximated ISI at the Receiver for a 16QAM Signal Sent via a FIR Channel Based Only on the Received Information and Statistical Techniques ${ }^{\dagger}$
}

\author{
Monika Pinchas and Hagar Turgeman \\ Ariel University, Ariel, Israel \\ † Presented at the Entropy 2021: The Scientific Tool of the 21st Century, 5-7 May 2021; Available online: \\ https://sciforum.net/conference/Entropy2021/.
}

Published: 5 May 2021

Let us consider for a moment the digital communication case where during transmission, a source signal undergoes a convoluted distortion between its symbols and the channel impulse response. This distortion is referred to as inter-symbol interference (ISI) which causes harmful distortions and presents a major difficulty in the recovery process. A Single-input-multiple-output (SIMO) channel is obtained from the use of an array of antennas in the receiver where the same information is transmitted through different sub-channels, all received sequences will be distinctly distorted versions of the same message. The ISI level from each sub-channel is unknown up to now to the receiver. Thus, even when one or more sub-channels cause heavy ISI, the whole information from all the sub-channels was still considered in the receiver. Obviously, if we know the approximated ISI of each sub-channel, we would use in the receiver only those sub-channels with the lowest ISI level to get improved system performance. In this talk we present a systematic way for getting the approximated ISI from each subchannel modeled as a finite-impulse-response (FIR) channel with real-valued coefficients for a 16QAM (16 quadrature amplitude modulation, a modulation using $\pm\{1,3\}$ levels for in-phase and quadrature components) source signal transmission. The approximated ISI is based on the Maximum Entropy density approximation technique, on the Edgeworth Expansion up to order six, on the Laplace integral method and on the generalized Gaussian distribution (GGD). Although the approximated ISI was derived for the noiseless case, it was tested successfully for signal to noise ratio (SNR) of SNR $=30 \mathrm{~dB}$ and $\mathrm{SNR}=20 \mathrm{~dB}$ as well.

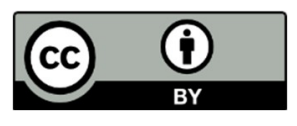

(C) 2021 by the authors. Licensee MDPI, Basel, Switzerland. This article is an open access article distributed under the terms and conditions of the Creative Commons Attribution (CC BY) license (http://creativecommons.org/licenses/by/4.0/). 